\title{
Recurrent Cryptococcosis in a Human Immunodeficiency Virus-negative Patient
}

\author{
Lillian C. Man, MD \\ Acknowledgements: Dr. Jeffrey B. Baliff, Jefferson University Department of Pathology
}

\section{INTRODUCTION}

Idiopathic CD4+ lymphocytopenia (ICL) is a rare disorder that can predispose otherwise immunocompetent individuals to life-threatening opportunistic infections. We present a case of a human immunodeficiency virus (HIV)-negative patient with ICL who presented with recurrent cryptococcosis.

\section{CASE PRESENTATION}

A 39-year-old Caucasian, HIV-negative male with a past medical history of stroke 13 years prior to admission and an episode of cryptococcal meningitis 18 months prior to admission presented to the hospital with generalized weakness for 7 days. The patient had a 10 pack-year smoking history and no significant environmental or occupational exposures. On admission, vital signs were significant for a temperature of $101.1^{\circ} \mathrm{F}$, a heart rate of 117 beats per minute, and a blood pressure 93/75 mm $\mathrm{Hg}$. Physical exam was remarkable for an ill-appearing male with dry mucous membranes and tachycardia.

Initial laboratory studies were significant for a white blood count of 3.14 cells $/ \mu \mathrm{L}$ (normal range $=4.5-11$ cells $/ \mu \mathrm{L}$ ), hemoglobin of $8.7 \mathrm{~g} / \mathrm{dL}$ (normal range $=13.5-17.5 \mathrm{~g} /$ $\mathrm{dL}$ ), and creatinine of $5.2 \mathrm{mg} / \mathrm{dL}$ (normal range $=0.6-1.2$ $\mathrm{mg} / \mathrm{dL}$ ). Blood cultures were positive for Cryptococcus neoformans. Subsequent laboratory studies revealed a serum cryptococcal antigen titer of 1:256 and a CD4+ count of $227 \mathrm{cells} / \mathrm{\mu L}$ (normal range $=410-1590$ cells $/$ $\mu \mathrm{L})$. The CD4+ count was 98 cells $/ \mu \mathrm{L}$ when the patient was diagnosed with cryptococcal meningitis 18 months prior. Enzyme-linked immunosorbent assays for HIV were negative. Anti-nuclear antibody titer was 1:80 and anti-double stranded DNA, anti-Smith, anti-SSA, anti-SSB and anti-ribonucleoprotein antibodies were negative. Lumbar puncture (LP) showed a normal opening pressure and was negative for cryptococcal infection.

Chest $X$-ray revealed a right pleural mass. Subsequent CT of the thorax revealed a $6.3 \times 3.3 \mathrm{~cm}$ pleural-based

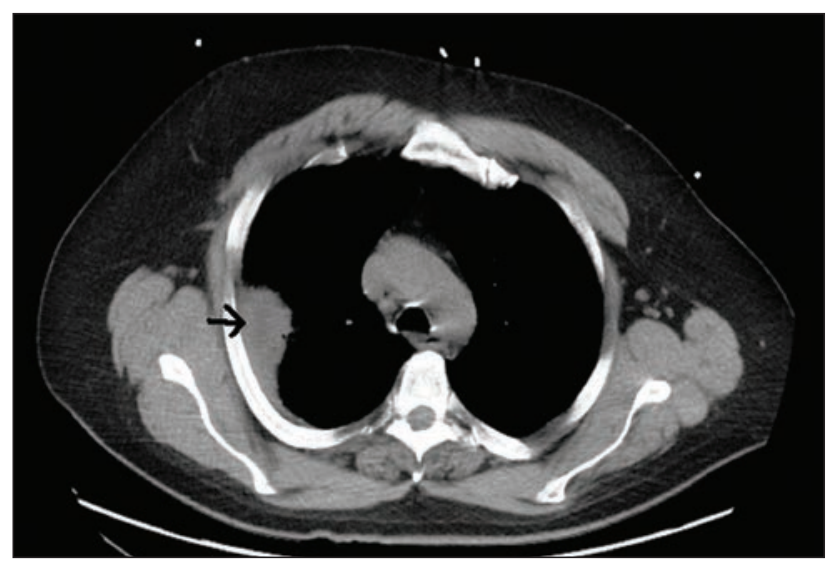

Figure 1: CT thorax without contrast showing a right pleural mass (arrow)

mass in the right upper lobe of the lung (Figure 1). Due to suspicion for a pulmonary malignancy, a Technetium bone scan was performed and found increased uptake in the right femur and left humerus. X-ray of the right femur showed well-circumscribed lytic lesions in the mid-femoral diaphysis (Figure 2). CT-guided biopsy of the pleural mass and the femur lesion revealed narrow-based, budding yeast that stained Gomori methenamine silver (Figure 3a) and a capsule that stained red with mucicarmine (Figure 3b), consistent with a diagnosis of cryptococcosis

\section{OUTCOME AND FOLLOW-UP}

The patient was treated with a four-week course of liposomal amphotericin B and flucytosine and then discharged on fluconazole. He was also recommended to follow up at the National Institutes of Health $(\mathrm{NIH})$ for further work-up of ICL as the predisposing etiology of his recurrent cryptococcosis.

Two months after discharge, the patient was hospitalized at an outside hospital for muscle pain and 
Figure 2: X-ray of right femur. Lytic lesions are shown (circled)

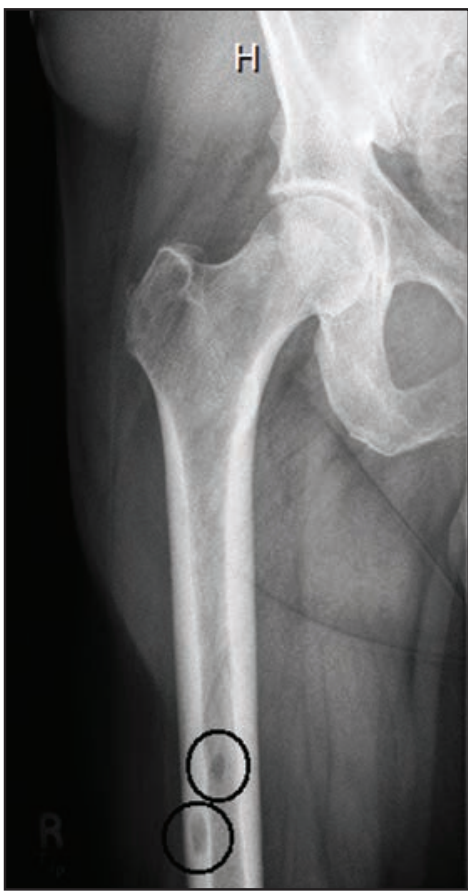

was still undergoing evaluation. He had not followed up at the NIH yet and was still on fluconazole therapy.

\section{DISCUSSION}

ICL is a rare disorder first defined by the US Centers for Disease and Control in 1992 as a CD4+ count $<300$ cells $/ \mathrm{mm}^{3}$ or a CD4+ cell count $<20 \%$ of total T-cells on two occasions and no serologic evidence of HIV infection. ${ }^{1}$ The pathogenesis of $I C L$ remains unclear. Researchers suggest a multifactorial etiology from diminished T-cell precursors, increased CD4+ lymphocyte apoptosis, and genetic factors. ${ }^{2,3}$ There is also an association between autoimmune diseases and ICL, most commonly Sjogren's. However the mechanism by which autoimmunity contributes to ICL's pathogenesis is ill-defined. ${ }^{2}$ Some reports suggest IL-2 therapy may increase CD4+ counts as well as decrease susceptibility to opportunistic infections, but currently there are no clear strategies to increase CD4+ lymphocytes. 3

The most important differential diagnosis for ICL is HIV infection. ICL typically becomes apparent through the manifestation of opportunistic infections, the most prevalent of which are cryptococcosis, mycobacteriosis, and herpes zoster. ${ }^{4}$ Disseminated cryptococcosis

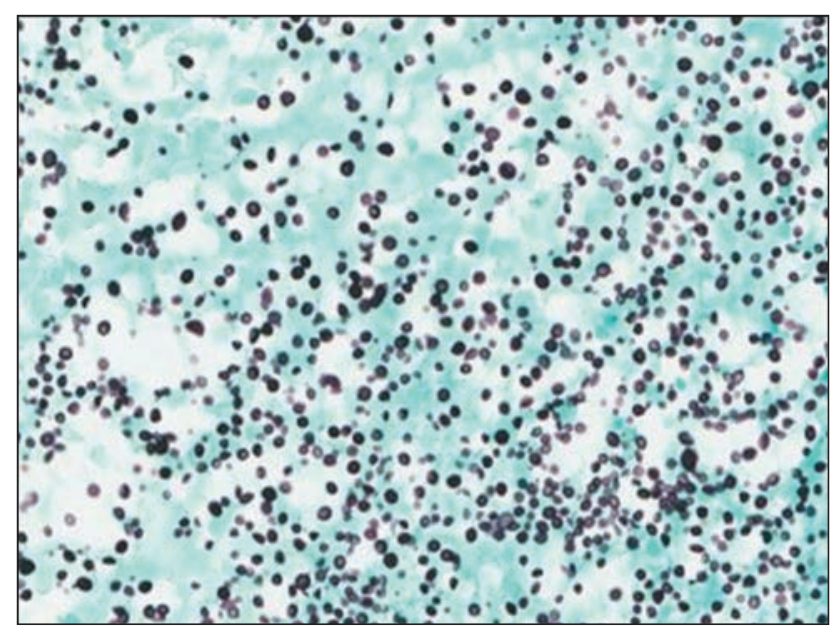

Figure 3a: Gomori methenamine silver (GMS) of the pleural mass biopsy showing innumerable darkly stained yeast.

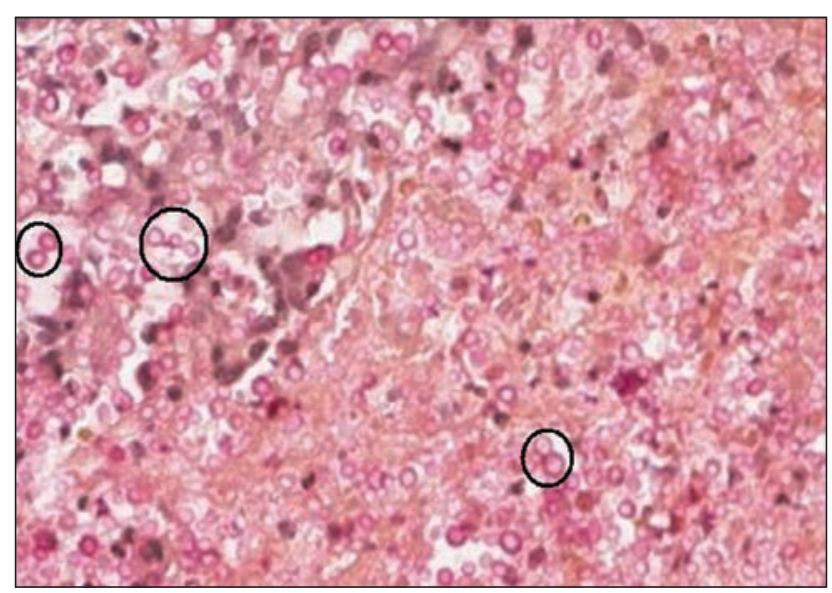

Figure 3b: Mucicarmine stain of the pleural mass biopsy. Multiple budding yeast are shown (circled).

can affect multiple organ systems including the adrenal glands, prostate, and bones and may be mistaken for malignancy with metastasis, ${ }^{5}$ as was the case with our patient. Given that meningitis is the most common presenting illness in patients with cryptococcosis, LP is essential even in the absence of neurological symptoms. ${ }^{6.7}$ Untreated cryptococcal meningitis carries a high mortality rate and can lead to cranial nerve palsies, hearing loss, and blindness. ${ }^{8}$

According to the current Infectious Diseases Society of America guidelines, non-HIV patients with disseminated cryptococcosis should be treated with a four-week course of amphotericin B and flucytosine for induction therapy followed by maintenance therapy with

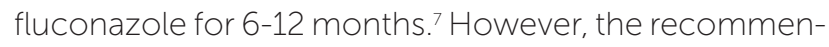


dations for non-HIV patients with cryptococcosis are often based on studies of HIV-infected patients. Given that our patient presented with recurrent cryptococcosis in the presence of $I C L$, it was thought that he should be maintained on fluconazole indefinitely.

\section{KEY POINTS}

In summary, ICL patients can present with an array of opportunistic infections even in the absence of HIV. The pathogenesis of $I C L$ remains unclear, and guidelines on the treatment of ICL patients with opportunistic infections are largely based on studies of HIV-infected patients. Cryptococcosis is one of the most common opportunistic pathogens that infect ICL patients. Patients with cryptococcosis should have an LP even in the absence of neurologic symptoms due to the high morbidity and mortality of untreated cryptococcal meningitis.

\section{REFERENCES}

1. Smith DK, Neal JJ, Holmberg et al. Unexplained opportunistic infections and CD4+ T-lymphocytopenia without HIV infection. N Engl J Med. 1993; 328(6): 374-379

2. Ahmad DS, Esmadi M, Steinmann WC. Idiopathic CD4 lymphocytopenia: Spectrum of opportunistic infections, malignancies, and autoimmune diseases. Avicenna J Med. 2013; 3(2): 37-47

3. Luo L, Li T. Idiopathic CD4 lymphocytopenia and opportunistic infection - an update. FEMS Immunol Med Microbiol. 2008; 54: 283-289.

4. Walker UA, Warnatz K. Idiopathic CD4 lymphocytopenia. Curr Opin Rheumatol. 2006; 18: 389-395.

5. Perfect J. Cryptococcus neoformaons. In: Mandell GL, Bennett JE, Dolin R. Mandell, Douglas, and Bennett's Principles and Practice of Infectious Diseases. 7th ed. Philadelphia, PA: Churchill Livingstone; 2009. pp 3287-3303.

6. Kiertiburanakul S, Wirojtananugoon S, Pracharktam R, Sungkanuparph S. Cryptococcosis in human immunodeficiency virus-negative patients. Int J Infect Dis. 2006; 10: 72-78.

7. Perfect JR, Dismukes WE, Dromer $F$ et al. Clinical practice guidelines for the management of cryptococcal disease: 2010 update by the Infectious Diseases Society of America. Clin Infect Dis. 2010; 50: 291-322.

8. Saag MS, Graybill RJ, Larsen RA, et al. Practice guidelines for the management of cryptococcal disease. Clin Infect Dis. 2000; 30: 710-718.

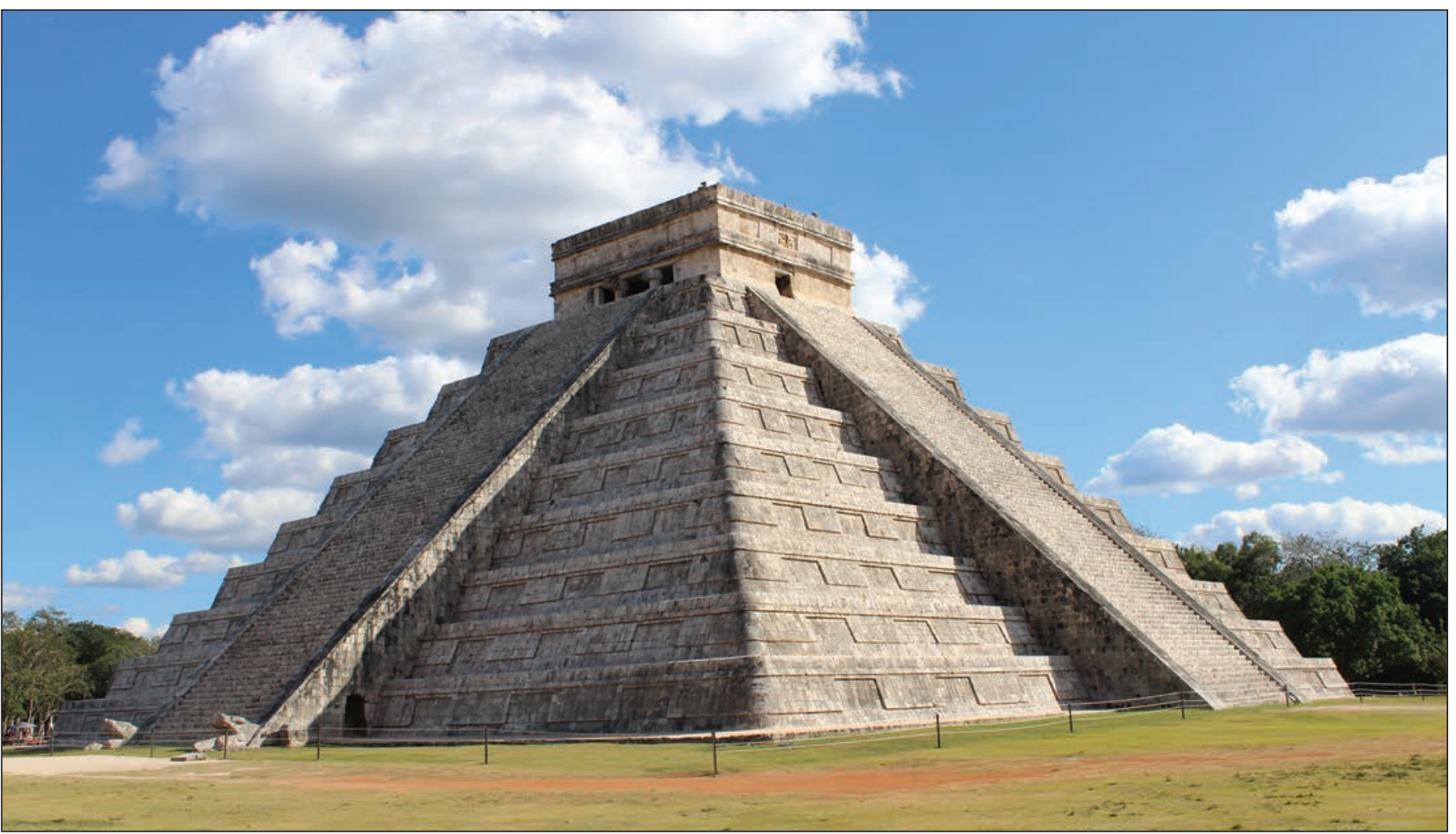

